\section{Draft Genome Resource of Fusarium oxysporum f. sp. capsici, the Infectious Agent of Pepper Fusarium Wilt}

\author{
Ping Xingxing, ${ }^{1}$ Raja Asad Ali Khan, ${ }^{1,2}$ Li Yan, ${ }^{1}$ Yang Yuhong, ${ }^{1}$ Xie Bingyan, ${ }^{1}$ Mao \\ Zhenchuan, ${ }^{1}$ and Ling Jian ${ }^{1, \dagger}$ \\ ${ }^{1}$ Institute of Vegetables and Flowers, Chinese Academy of Agricultural Sciences, Beijing 100081, \\ China \\ ${ }^{2}$ Department of Plant Pathology, The University of Agriculture Peshawar, 25000, Pakistan
}

\begin{abstract}
Fusarium oxysporum f. sp. capsici is the specific pathogen of pepper Fusarium wilt and causes a significant reduction in pepper yield. Its narrow host specificity has led to the concept of formae speciales. This interesting phenomenon has great potential and needs to be analyzed at the molecular level. In this study, we obtained the draft genome sequence of $F$. oxysporum f. sp. capsici, using the Oxford Nanopore sequencing technology. The long read-based assembly consisted of 34 contigs, with a total length of $54,516,562 \mathrm{bp}$. The contig $\mathrm{N}_{50}$ was $4,962,668 \mathrm{bp}$ and the $\mathrm{GC}$ content was $47.6 \%$. Our genome assembly of $F$. oxysporum f. sp. capsici provides a valuable resource for the study of pepper Fusarium wilt, and the comparative genomic study of $F$. oxysporum.
\end{abstract}

\section{Genome Announcement}

Fusarium oxysporum is a filamentous fungus that is a soilborne pathogen of plants and an opportunistic pathogen of humans. Moreover, F. oxysporum is considered to be a complex species with a broad host range, and its host specificity can be used for classification into various formae speciales (ff. spp.) (Ma et al. 2013). Fusarium oxysporum f. sp. capsici infects pepper plants and causes wilting, which results in heavy production losses in China.

Many F. oxysporum genomes have been sequenced, with most of them sequenced using the short reads of Illumina sequencing technology, resulting in a highly fragmented genome assembly (Wang et al. 2020; Ye et al. 2011). Highly fragmented genomes are not conducive to structural resolution. The third-generation platform from Oxford Nanopore Technologies (ONT) is able to generate ultra-long read lengths. Several hundred kilobases can be reached, resulting in more contiguous genomes being acquired and facilitating the analysis of genome structural variants (De Maio et al. 2019; Jain et al. 2018).

In June 2014, the F. oxysporum f. sp. capsici strain was isolated from a susceptible pepper-HaiJiao 4-in Putian City, Fujian Province, China. The pathogen was identified morphologically and through an internal transcribed sequencing analysis. The pathogenicity was confirmed by Koch's postulates. In this study, we extracted high-molecular weight genomic DNA of $F$. oxysporum f. sp. capsici from a pure culture, using a Qiagen genomic DNA

${ }^{\dagger}$ Corresponding author: L. Jian; lingjian@ caas.cn

*The $e$-Xtra logo stands for "electronic extra" and indicates there is supplementary material published online.

The author(s) declare no conflict of interest.

Accepted for publication 27 January 2021.

\section{Funding}

This research is funded by the National Key R\&D Projects

(2018YFD0201204), National Natural

Science Foundation of China

(31571962), Key Laboratory of Biology and Genetic Improvement of Horticultural Crops, Ministry of Agriculture, P.R. China. China Agriculture Research System (CARS-25).

\section{Keywords}

Fusarium oxysporum f. sp. capsici, Fusarium wilt, genome, pepper 
Table 1. Assembly statistics of the Fusarium oxysporum f. sp. capsici

\begin{tabular}{lc} 
Feature & Statistics \\
Genome [bp] & $54,516,562$ \\
Read length $N_{50}$ & 15,656 \\
Mean read length[bp] & $11,037.4$ \\
Maximum read length & 143634 \\
Number of contigs & 34 \\
Largest contigs [bp] & $7,838,233$ \\
Contig N $N_{50}[\mathrm{bp}]$ & $4,962,668$ \\
GC & $47.6 \%$ \\
Assemble completeness (FGMP) & $93.5 \%$ \\
National Center for Biotechnology Information accession & JABCSD000000000 \\
\hline
\end{tabular}

extraction kit. The sequencing library was prepared using a ligation sequencing kit in accordance with the ONT standard protocol. For sequencing, flow cells version and pore version R10.3 were used on the PromethION device.

According to the data, we obtained $1,058,257$ reads, which is about $11.68 \mathrm{~Gb}$ bases (approximately 200x genome coverage). Overall, the mean read length was 11,037.4 bp and the read length $\mathrm{N}_{50}$ value was $15,656 \mathrm{bp}$. We assembled the genome using Canu version 1.6 (Koren et al. 2017) and polished the initial assembly based on Nanopore reads using Racon version 1.4.3, with the default parameters (Vaser et al. 2017) (Table 1). All 35 contigs were compared with the mitochondrial genome MF155191.1 (Zeng et al. 2017), and only tig00000113 had a high similarity with mitochondrial sequences. Based on gene models from Fusarium graminearum, we predicted repeat sequences and protein-encoding genes from the final assembly, using RepeatMasker version 4.0.7, based on the Repbase fungi data set (Chen 2004), and AUGUSTUS version 2.7 (Stanke et al. 2004), respectively. Using AUGUSTUS, the predicted number of protein-encoding genes was 16,618 . The total size of the interspersed repeats was $1,875,337 \mathrm{bp}$, accounting for $3.44 \%$ of the $F$. oxysporum $\mathrm{f}$. sp. capsici genome. Furthermore, we used FGMP version 1.0.2 (Cissé and Stajich 2019) to evaluate the assembly quality and found $93.5 \%$ completeness.

The F. oxysporum species complex has received substantial attention from scientists for more than a century because of its economic losses and wide host range. The narrow host specificity of pathogenic strains has led to the concept of formae speciales. This interesting phenomenon has great potential and needs to be analyzed at the molecular genetics level. Various forms of draft genome resources have a wide range of potential uses. The draft genome resource presented in this study is the first reported genome of $F$. oxysporum $\mathrm{f}$. sp. capsici and will provide a fundamental resource for the investigation of the pathogenic mechanism of this strain. In addition, it will also be useful for the study of the comparative genome of $F$. oxysporum. This Whole Genome Shotgun project has been deposited at DDBJ/ENA/ GenBank under the accession JABCSD000000000. The version described in this paper is version JABCSD000000000.

\section{Acknowledgments}

We thank X. Rongfeng from the Fujian Agriculture and Forestry University for providing the F. oxysporum f. sp. capsici strain.

\section{Literature Cited}

Chen, N. 2004. Using RepeatMasker to identify repetitive elements in genomic sequences, Chapter 4. Curr. Protoc. Bioinformatics.

Cissé, O. H., and Stajich, J. E. 2019. FGMP: Assessing fungal genome completeness. BMC Bioinformatics 20:184.

De Maio, N., Shaw, L. P., Hubbard, A., George, S., Sanderson, N. D., Swann, J., Wick, R., AbuOun, M., Stubberfield, E., Hoosdally, S. J., Crook, D. W., Peto, T. E. A., Sheppard, A. E., Bailey, M. J., Read, D. S., Anjum, M. F., Walker, A. S., and Stoesser, N. 2019. On behalf of the rehab consortium.
Comparison of long-read sequencing technologies in the hybrid assembly of complex bacterial genomes. Microb. Genom. 5:e000294.

Jain, M., Koren, S., Miga, K. H., Quick, J., Rand, A. C., Sasani, T. A., Tyson, J. R., Beggs, A. D., Dilthey, A. T., Fiddes, I. T., Malla, S., Marriott, H., Nieto, T., O'Grady, J., Olsen, H. E., Pedersen, B. S., Rhie, A., Richardson, H., Quinlan, A. R., Snutch, T. P., Tee, L., Paten, B., Phillippy, A. M., Simpson, J. T., Loman, N. J., and Loose, M. 2018. Nanopore sequencing and assembly of a human genome with ultra-long reads. Nat. Biotechnol. $36: 338-345$. 
Koren, S., Walenz, B. P., Berlin, K., Miller, J. R., Bergman, N. H., and Phillippy, A. M. 2017. Canu: Scalable and accurate long-read assembly via adaptive k-mer weighting and repeat separation. Genome Res. 27:722-736.

Ma, L. J., Geiser, D. M., Proctor, R. H., Rooney, A. P., O'Donnell, K., Trail, F., Gardiner, D. M., Manners, J. M., and Kazan, K. 2013. Fusarium pathogenomics. Annu. Rev. Microbiol. 67:399-416.

Stanke, M., Steinkamp, R., Waack, S., and Morgenstern, B. 2004. AUGUSTUS: A web server for gene finding in eukaryotes. Nucleic Acids Res. 32 (Web Server):W309-W312.

Vaser, R., Sović, I., Nagarajan, N., and Sikić, M. 2017. Fast and accurate de novo genome assembly from long uncorrected reads. Genome Res. 27:737-746.
Wang, B., Yu, H., Jia, Y., Dong, Q., Steinberg, C., Alabouvette, C., Edel-Hermann, V., Kistler, H. C., Ye, K., Ma, L. J., and Guo, L. 2020. Chromosomescale genome assembly of Fusarium oxysporum strain Fo47, a fungal endophyte and biocontrol agent. Mol. Plant-Microbe Interact. 33:1108-1111.

Ye, L., Hillier, L. W., Minx, P., Thane, N., Locke, D. P., Martin, J. C., Chen, L., Mitreva, M., Miller, J. R., Haub, K. V., Dooling, D. J., Mardis, E. R., Wilson, R. K. Weinstock, G. M., and Warren, W. C. 2011. A vertebrate case study of the quality of assemblies derived from next-generation sequences. Genome Biol. 12:R31.

Zeng, L., Liu, C., Lin, R., Kang, X., Xie, B., and Xiong, X. 2017. Complete mitogenome of the high ethanol production fungus Fusarium oxysporum Mh2-2. Mitochondrial DNA B Resour. 2:814-815. 